\begin{tabular}{c|c|c}
\hline \hline & $a-x$ & $k_{1}$ \\
\hline 30 & 8,67 & 0,00207 \\
74,6 & 7,1 & 0,00199 \\
167 & 4,6 & 0,00202 \\
318,6 & 2,7 & 0,00179
\end{tabular}

Fơr $w_{m}=0,052$ ist also die mittlere Konstante 0,00197 , fâr $c=0,10,0013^{2}$ gegen 0,00094, unkorr. 0,00098 (w $\left.w_{m}=0,05^{8}\right)$ bei Kailan.

Christiania, April I909.

(Eingegangen: 6. April.)

\title{
KINETIK DER UMSETZUNG VON ALKYLSULFATEN MIT ANORGANISCHEN SALZEN.
}

Von $P$. Walden und M. Centnerszwer.

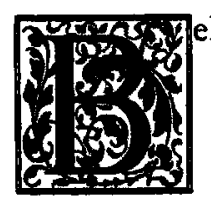
ekanntlich haben das Dimethyl- und Diathylsulfat wegen ihrer großen Reaktionsfahigkeit mannigfache Verwendung sowohl in der Laboratoriumspraxis als auch in der chemischen Technik gefunden. Besonderes Interesse verdienen z. B. die Umsetzungen dieser Ester mit Metallhalogeniden ${ }^{1}$, sowie mit den anorganischen Rhodaniden, Cyaniden und Nitriten ${ }^{2}$ ); die hier auftretenden Reaktionen zeichnen sich durch Einfachbeit, schnellen Verlauf und gute Ausbeuten aus. Infolgedessen hat schon seinerzeit der eine von uns die physikalisch - chemische Untersuchung dieser Reaktionen angemeldet ${ }^{3}$ ). Als erstes Ergebnis dieser umfangreichen Studien erscheint nun die vorliegende Mitteilung. Es galt, zuerst die Reaktionsordnung sowie die verschiedenen Einflasse zu ermitteln, welche die Geschwindigkeit der Umsetzung beider Ester beeinflussen.

Das nachfolgende Zahlenmaterial bezieht sich zunächst auf das Dimethylsulfat $\left(\mathrm{CH}_{3}\right)_{2} \mathrm{SO}_{4}$, welches mit den Halogeniden und Rhodaniden in Wechselwirkung gebracht wurde, und zwar mit Natriumjodid $\mathrm{NaJ}$, Cadmiumjodid $\mathrm{Cd} J_{2}$, Zinnjodid $\mathrm{Sn} J_{4}$ und Kaliumbromid $\mathrm{KBr}$, ferner mit Natrium-, Kalium- und Ammoniumrhodanid $\mathrm{NaCNS}, \mathrm{KCNS}, \mathrm{NH}_{4} \mathrm{CNS}$, sowie mit Kobaltrhodanid $\mathrm{Co}(\mathrm{CNS})_{2}$. Als Lösungsmittel wurde Methylalkohol (in einzelnen Ver. suchsreihen auch Aceton) gewählt; hierfar war der Umstand bestimmend, daß die Geschwindigkeitsmessungen in einem homogenen Mittel vollzogen werden sollten. Nun lost sich Dimethylsulfat in Wasser wenig und erleidet darin eine Hydrolyse, ebenso schwerloslich sind die Endprodukte (Alkyljodid und - bromid sowie Alkylrhodanid): dagegen lost Methylalkohol sowohl das Methylsulfat als auch die Meistzahl der zur Umsetzung gebrachten Halogen- und (1905).

I) Weinland und Schmidt, Berl. Ber. 38, 2327

2) Kaufler und Pomeranz, Sitz.-Ber. d. Wien. Akad. 110, II (190I); Walden, Berl. Ber. 40, $32{ }^{2} 4$ (1907).

3) Walden, 1. c. 3217
Rhodanmetalle sowie die gebildeten Halogenund Rhodanalkyle.

Untersuchungsmethode. $\mathrm{Da}$ bei den vorliegenden Reaktionen das Halogen- und Rhodanion aus der Lösung verschwindet und mit dem Methyl des Dimethylsulfats zu Halogenund Rhodanmethyl sich vereinigt, so ließ sich das Fortschreiten der Umsetzung sehr bequem durch Titration mit Silbernitratlosung verfolgen. Es wurde einerseits eine methylalkoholische (bezw. Aceton-) Lossung des Dimethylsulfats, andererseits eine Lősung des gewanschten Metallsalzes in Methylalkohol (bezw. Aceton) von bestimmter Konzentration hergestellt, einzeln auf die entsprechende Versuchstemperatur gebracht, alsdann im gegebenen Moment zusammengegossen und gleichzeitig durchmischt. In passenden Zeitintervallen wurde nun aus dem im Thermostaten befindlichen, gut verschlossenen Glaskölbchen eine Probe des Reaktionsgemisches herauspipettiert und in überschüssige Silbernitratlösung entleert. Durch Zuracktitrieren mit Rhodanammoniumlosurg nach Volhard wurde die noch vorhandene Menge der Halogen - bezw. Rhodanionen des entnommenen Reaktionsgemisches bestimmt. Der Endwert des Titers bei der Reaktion wurde gewöhnlich nach einem Tage bestimmt und dann durch nachfolgende Proben kontrolliert. Die Silbernitrat - und Rhodanammoniumlosungen waren gewöhnlich $1 / 20$ n. ${ }^{1}$ ).

Die Rolle der Temperatur wurde durch Geschwindigkeitsmessungen bei $0,12,6$ bezw. 25 und $40^{\circ} \mathrm{C}$. ermittelt. Der Einfluß der Natur der Metalle, die mit dem Halogen und Rhodan verbunden waren, wurde durch die Verwendung der obengenannten Alkali-, Ammonium-, Cadmium -, Kobalt - und Zinnsalze studiert. Zwecks Bestimmung der Rolle der Konzentration und der molekularen Verhăltnisse beider reagierenden Körper wurden sowohl aquivalente Mengen als auch ein Ueber-

1) Bei der Ausfthinrung dieser Messungen haben uns die Herren stud. chem. M. Cholodkowsky und stud. chem. P. Grebnew eifrigst Dienste geleistet. 
schub bald des Dimethylsulfats, bald des Salzes verwandt.

Die Reinheit der Reaktionsprodukte sowie der Losungsmittel wurde sorgfaltig gewahrt. Die Salze wurden (meist aus wasserfreiem Methylalkohol) umkristallisiert, im elektrisch geheizten Vakuumexsikkator getrocknet und durch Titration analysiert; das Dimethylsulfat (Kahlbaum) wurde im Vakuum fraktioniert destilliert, und der Methylalkohol - scharf aber Aetzkalk getrocknet - innerhalb I 0 herausdestilliert.

\section{Die Reaktionsordnung.}

Bezeichnen wir mit $\mathrm{Me}$ die Metalle $\mathrm{K}, \mathrm{Na}$ (bezw. $\mathrm{NH}_{4}$ ) $, \frac{\mathrm{Cd}}{2}, \frac{\mathrm{Co}}{2}$ und $\frac{S n}{4}$, so haben wir: bezw.$$
\left(\mathrm{CH}_{8}\right)_{2} \mathrm{SO}_{4}+\mathrm{MeJ}=\mathrm{CH}_{3} \mathrm{~J}+\mathrm{MeCH}_{3} \mathrm{SO}_{4}
$$$$
\left(\mathrm{CH}_{3}\right)_{2} \mathrm{SO}_{4}+\mathrm{Me} \mathrm{CNS}=\mathrm{CH}_{3} \mathrm{CNS} \text { (Methyl- }
$$
rhodanid) $+\mathrm{MeCH}_{3} \mathrm{SO}_{4}$.

Diese beiden Reaktionsgleichungen der studierten Umsetzungen, welche zunächst ohne Rücksicht auf die elektrolytische Dissoziation der Komponenten und unter der Annahme der Bildung des Methylrhodanids geschrieben werden, lassen erwarten, dab wir es hier mit einer bimolekularen Reaktion zu tun haben. Dann ist - im Fall ăquimolekularer Lösungen beider Bestandteile - die Geschwindigkeitskonstante ${ }^{1}$ :

$$
K=\frac{a_{0}-a_{n}}{\left(a_{n}-a_{\infty}\right) a_{0} t} \cdot \frac{v}{n}
$$

In der Formel für $K$, wie auch in den weiteren Tabellen und Formeln haben die Buchstaben folgende Bedeutung:

$a_{o}$ Anfangstiter,

$a_{n}$ Titer zur Zeit $t$ in Minuten,

$a_{\infty}$ Endtiter,

$\stackrel{v}{ }$ das zum Titrieren herausgenommene Volum des Reaktionsgemisches (in Kubikzentimetern),

$n$ die Normalitat der zum Titrieren benutzten Silbernitratlösung (in Molen pro Liter).

Ueber die Anwendbarkeit der Formel (I) auf die studierten Reaktionen geben die folgenden Tabellen Aufschluf; in allen diesen Tabellen ist unter "Anfangskonzentration" die molare Konzentration der Lösungen vor dem Ver. mischen angegeben.

Die tabellierten Werte für $a$ sind Mittelwerte aus mehreren Versuchsreihen. Der Endwert $a$ $=\mathrm{r}, 3$ für $t=\infty$ zeigt nun, dafi die Reaktion wohl aber $90 \%$ Umsatz gibt, jedoch nicht vollständig zu Ende geht. Die Konstanz der

I) Ostwald, Lehrb. d. allgem. Chemie 2 [2], 212, Leipzig 1897; Nernst, Theoret. Chemie, S. 552, Stuttgart 1907 .
Tabelle I.

$\left(\mathrm{CH}_{3}\right)_{8} \mathrm{SO}_{4}+\mathrm{NaJ}-\mathrm{CH}_{3} \mathrm{~J}+\mathrm{CH}_{3} \mathrm{NaSO}_{4}$. Lösungsmittel: Methylalkohol. Temperatur: 0 . $n=1 / 20 . \quad v=2 \mathrm{ccm}$.

\begin{tabular}{|c|c|c|c|c|c|}
\hline \multicolumn{3}{|c|}{$1 / 1$ n. $\left(\mathrm{CH}_{3}\right)_{2} \mathrm{SO}_{4}+1 / 1$ n. $\mathrm{NaJ}$} & \multicolumn{3}{|c|}{$1 / 8$ n. $\left(\mathrm{CH}_{3}\right)_{8} \mathrm{SO}_{4}+1 / 8$ n. $\mathrm{NaJ}$} \\
\hline$t$ & $a$ & $\boldsymbol{K}$ & $t$ & $a$ & $\boldsymbol{K}$ \\
\hline 0 & $20,0 \mathrm{ccm}$ & - & 0 & $6,34 \mathrm{ccm}$ & - \\
\hline Io & $\mathrm{I} 7,5$, & 0,0308 & ro & $6,22, "$ & 0,0362 \\
\hline 20 & 15,8, & 0,0290 & 20 & $6,08 \ldots$ & 0,0404 \\
\hline 50 & $\mathrm{I} 2,2 "$ & 0,0286 & $5^{\circ}$ & $5,77 "$ & 0,0377 \\
\hline 100 & 8,8 & 0,0298 & Ioo & $5,3^{\circ} "$ & 0,0381 \\
\hline I50 & 6,8, & 0,0320 & I50 & 4,92 & $0,0 \mathbf{3}^{81}$ \\
\hline 200 & 6,0, & 0,0298 & 200 & 4,53 & 0,0405 \\
\hline $25^{\circ}$ & $5.4 "$ & 0,0285 & 400 & 3,60 & 0,0416 \\
\hline$\infty$ & 1,3 & - & $\infty$ & $\mathbf{I}, \infty 0$ & - \\
\hline \multicolumn{3}{|c|}{$K_{\text {mittel }}=0,0298$} & \multicolumn{3}{|c|}{$K_{\text {mittel }}=0,03^{89}$} \\
\hline
\end{tabular}

Tabelle 2.

$\left(\mathrm{CH}_{3}\right)_{2} \mathrm{SO}_{4}+\mathrm{NaCNS}=\mathrm{CH}_{3} \mathrm{CNS}+\mathrm{CH}_{3} \cdot \mathrm{NaSO}_{4}$. Lösungsmittel: Methylalkohol. Temperatur: $25^{\circ}$. $n-1 / 20 . \quad v=2 \mathrm{ccm}$.

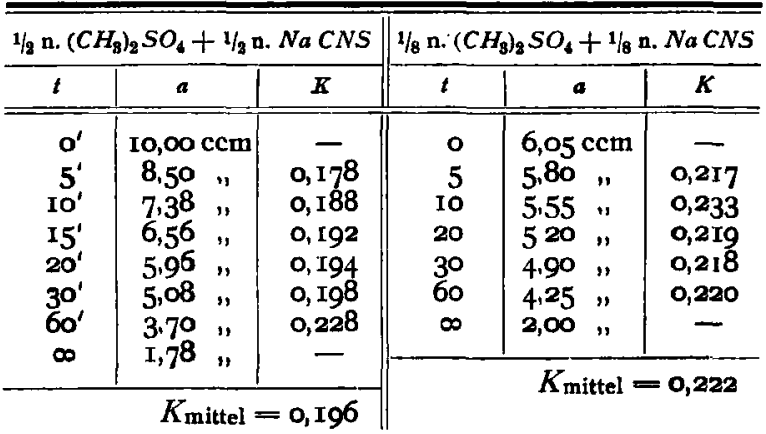

$K$-Werte kann als befriedigend angesehen werden, sie beweist, daß die Reaktion zwischen Dimethylsulfat 'und Natriumjodid tatsächlich nach dem obigen Schema:

$\left(\mathrm{CH}_{3}\right)_{2} \mathrm{SO}_{4}+\mathrm{NaJ}=\mathrm{CH}_{3} \mathrm{~J}+\mathrm{NaCH}_{3} \mathrm{SO}_{4}$, d. h. bimolekular verlauft. Dasselbe labt sich auch in bezug auf die andere Reaktion feststellen.

Die Reaktion zwischen Dimethylsulfat und Metallrhodanid ist also ebenfalls als eine Reaktion zweiter Ordnung anzusprechen. $Z_{u}$ bemerken ist noch, daB auch hier die Umsetzung nicht bis zu Ende geht, und zwar ist der nichtumgesetzte Anteil beim Rhodanid noch gröBer, als beim Natriumjodid.

\section{Wechselnde Anfangskonzentration.}

$\mathrm{DaB}$ die vorliegenden Umsetzungen zu den bimolekularen Reaktionen gebören, wird unzweideutig erst dann festgestellt, wenn der Wert der Geschwindigkeitskonstante $K$ bei gleichbleibender Temperatur, trotz wechselnder Anfangskonzentration des Gemisches praktisch unveränderlich bleibt ${ }^{1}$ ). $\quad D a ß$ diese

I) Noyes, Zeitschr. f. physik, Chemie 18, II8 (I895). $40^{*}$ 
Forderung auch hinsichtlich der Reaktionen des Dimethylsulfats befriedigend erfallt wird, sollen die folgenden Zusammenstellungen dartun.

Tabelle 3.

$\left(\mathrm{CH}_{3}\right)_{2} \mathrm{SO}_{4}+\mathrm{NaJ}=\mathrm{CH}_{3} \mathrm{~J}+\mathrm{NaCH}_{3} \mathrm{SO}_{4}$.

Lösungsmittel: Methylalkohol. Temperatur: $0^{\circ} \mathrm{C}$.

\begin{tabular}{c|c|c}
\hline \multicolumn{2}{c|}{ Anfangskonzentration von } & $K=\frac{a_{0}-a_{n}}{\left(a_{n}-a_{\infty}\right) a_{0} t} \cdot \frac{v}{n}$ \\
\hline$\left(C H_{2}\right)_{2} S O_{4}$ & $N a J$ & 0,0298 \\
\hline $1 / 1 \mathrm{n}$. & $1 / 2 \mathrm{n}$. & 0,0295 \\
$1 / 2 \mathrm{n}$. & $1 / 2 \mathrm{n}$. & 0,0320 \\
$1 / 6 \mathrm{n}$. & $1 / 6 \mathrm{n}$. & 0,0389 \\
$1 / 8 \mathrm{n}$. & $1 / \mathrm{n}$. &
\end{tabular}

Tabelle 4.

$\left(\mathrm{CH}_{3}\right)_{2} \mathrm{SO}_{4}+\mathrm{NaCNS}=\mathrm{CH}_{2} \mathrm{CNS}+\mathrm{NaCH}_{3} \mathrm{SO}_{4}$.

Lösungsmittel: $\mathrm{CH}_{\mathrm{a}} \mathrm{OH}$. Temperatur: $25^{\circ} \mathrm{C}$.

\begin{tabular}{c|c|c}
\hline \multicolumn{2}{c|}{ Anfanggkonzentration von } & $K$ \\
\hline$\left(\mathrm{CH}_{\mathrm{g}} \mathrm{SO}_{4}\right.$ & $\mathrm{NaCNS}$ & \\
\hline $1 / 1 \mathrm{n}$. & $1 / \mathrm{n}$. & $0,19 \mathrm{I}$ \\
$1 / \mathrm{n}$. & $1 / \mathrm{n}$. & 0,196 \\
$1 / 4 \mathrm{n}$. & $1 / 4 \mathrm{n}$. & 0,223 \\
$1 / 8 \mathrm{n}$. & $1 / 8 \mathrm{n}$. & 0,222
\end{tabular}

Tabelle 5.

$\left(\mathrm{CH}_{3}\right)_{2} \mathrm{SO}_{4}+\mathrm{KCNS}-\mathrm{CH}_{3} \mathrm{CNS}+\mathrm{KCH}_{3} \mathrm{SO}_{4}$

Lösungsmittel: $\mathrm{CH}_{3} \mathrm{OH}$. Temperatur: $25^{\circ} \mathrm{C}$.

\begin{tabular}{|c|c|c|c|}
\hline \multicolumn{2}{|c|}{$\begin{array}{l}\text { Anfangskonzentration } \\
\text { von }\end{array}$} & \multirow[t]{2}{*}{$\boldsymbol{K}$} & \\
\hline$\left(\mathrm{CH}_{\mathrm{B}}\right)_{2} \mathrm{SO}_{4}$ & KCNS & & \\
\hline $\begin{array}{ll}1 / \mathrm{a} & \mathrm{n} . \\
1 / \mathrm{s} & \mathrm{n} . \\
1 / 8 & \mathrm{n} . \\
1 / 16 & \mathrm{n} .\end{array}$ & $\begin{array}{ll}1 / \mathrm{an} & \mathrm{n} \\
1 / \mathrm{n} & \mathrm{n} \\
1 / \mathrm{g} & \mathrm{n} . \\
1 / 1 \mathrm{n} & \mathrm{n} .\end{array}$ & $\begin{array}{l}0,224 \\
0,236 \\
0,299 \\
0,226\end{array}$ & $\begin{array}{l}\text { Die Schwerloslichkeit des } \\
\text { Kaliumrhodanids in Methyl- } \\
\text { alkohol verhinderte die Unter- } \\
\text { suchung normaler Losungen. } \\
\text { Beim Umsatz tritt allmuhlich } \\
\text { eine Ausscheidung des ge- } \\
\text { bildeten } K C H_{\mathrm{a}} S O_{4} \text { ein jedoch } \\
\text { auBerte sich dieser Umstand } \\
\text { nicht auf den Wert der Kon- } \\
\text { stante } K \text {. }\end{array}$ \\
\hline
\end{tabular}

Bemerkenswert ist die Tatsache, daB die Reaktionsgeschwindigkeit des Kalium rhodanids praktisch mit der des $\mathrm{Natriumrhodanids}$ zusammenfallt. Wie beim Natriumrhodanid (Tabelle 2), so bleibt auch beim Kaliumrhodanid nach Schluß der Reaktion ein meBbarer Anteil der einwirkenden Stoffe unzersetzt. Die Reaktion der Rhodansalze auf Dimethylsulfat ist eine unvollstăndige und umkehrbare. Der unveränderte Anteil des Rhodansalzes ist in allen Versuchen nahezu konstant und von der Anfangskonzentration unabhăngig, wie das vom Massenwirkungsgesetz gefordert wird. Dieser Anteil betrăgt:

beim Natriumrhodanid etwa $25 \%$

und beim Kaliumrhodanid " 20 " der angewandten Menge des Esters.

\section{Ueberschuß des einen Bestandteiles.}

Eine grundlegende Frage, welche sich an das Studium der Umsetzungen des Dimethyl- sulfats knüpft, lautet: Bleibt die Reaktion bei dem Ersatz einer Methylgruppe durch das Metall stehen oder ist auch die andere Methylgruppe des Dimethylsulfats reaktionsfähig? Der Versuch entschied diese Frage im ersten Sinne: Die Reaktion gebt nur bis zur Bildung des methylschwefelsauren Salzes.

Das geht deutlich aus Versuchen hervor, welche mit einem UeberschuB des Salzes angestellt wurden. Es wurden 2 Mole des Salzes mit I Mol des Esters vermischt; in allen Făllen blieb mehr als I Mol des Salzes unverändert zurück, wie die nachstehenden Zahlen beweisen:

\begin{tabular}{|c|c|c|}
\hline Anfangskonzentration & $\begin{array}{c}\text { Anfangstiter } \\
\mathbf{c c m}\end{array}$ & $\begin{array}{c}\text { Endtiter } \\
\mathrm{ccm}\end{array}$ \\
\hline $\begin{array}{llll}1 / 1 & \text { n. } N a J & 1 / 2 & \text { n. }\left(C H_{3}\right)_{2} \mathrm{SO}_{4} \\
1 / 1 & \text { n. } N a C N S & 1 / 2 & \text { n. } \\
1 / 2 & \text { n. KCNS } 1 / 4 & \text { n. }\end{array}$ & $\begin{array}{l}20 \\
20 \\
10\end{array}$ & $\begin{array}{r}11,5 \\
12,5 \\
5,5\end{array}$ \\
\hline
\end{tabular}

Ein Ueberschuf des Rhodanids verschiebt also das Gleichgewicht nach der Seite der Reaktionsprodukte, ganz im Sinne der Gleichgewichtslehre; im abrigen aber tritt immer nur ein Mol des Salzes auf ein Mol des Esters in Reaktion.

Bei der Berechnung der Reaktionsgeschwindigkeiten muB man in diesem Fall in Betracht ziehen, daB die Formel der Konstante wesentlich anders lautet, als bei aquivalenten Lösungen. Bezeichnen $a$ und $b$ die Anfangskonzentrationen des Salzes und des Esters (in Molen pro Liter) und $x$ die umgesetzte Menge beider in demselben $\mathrm{MaB}$, dann ist:

$$
\frac{d x}{d t}=K(a-x)(b-x) .
$$

Nach der Integration 1) erhält man den Ausdruck fur die Geschwindigkeitskonstante:

$$
K=\frac{1}{(a-b) t} \ln \frac{(a-x) b}{(b-x) a}
$$

Ist $a_{o}$ der Anfangstiter der Lösung in bezug auf das Salz, so ist $a=a_{o}$; ist weiter $a_{n}$ der Titer zur Zeit $t$, dann ist $x=a_{o}-a_{n_{1}}$ und ist schließlich $a_{\infty}$ der Schlußtiter, so können wir $b=a_{0}-a_{\infty}$ setzen. Dann erhalten wir die Gleichung:

Tabelle 6.

\begin{tabular}{|c|c|c|c|}
\hline $\begin{array}{l}\text { Tempe- } \\
\text { ratur } \\
\text { Grad }\end{array}$ & \multicolumn{2}{|c|}{ Anfangskonzentrationen } & $\frac{K}{\text { (nach Gleichung } 3)}$ \\
\hline $\begin{array}{r}0 \\
0 \\
0 \\
25 \\
25\end{array}$ & $\begin{array}{l}1 / 1 \text { n. } N a J \\
2 / 9 \text { n. " } \\
1 / 2 \text { n. } \\
1 / 1 \text { n. } N a C N \\
1 / \% \text { n. } K C N S\end{array}$ & 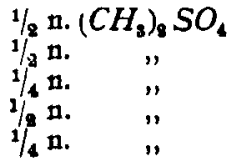 & $\begin{array}{ll}0,0309 & (0,0295) \\
0,0315 & (0,0295) \\
0,0299 & (0,0320) \\
0,173 & (0,196) \\
0,222 & (0,236)\end{array}$ \\
\hline
\end{tabular}

Lösungsmittel: Methylalkohol.

I) Nernst und Schönfließ, Einftuhrung in die mathematische Behandlung, I44 (I898). 
Tabelle 7 .

Lösungsmittel: Methylalkohol. Temperatur: $25^{\circ}$.

\begin{tabular}{|c|c|c|c|c|c|c|c|c|}
\hline \multirow{3}{*}{$\begin{array}{c}\text { Zeit } \\
\text { in } \\
\text { Minuten }\end{array}$} & \multicolumn{8}{|c|}{ Anfangsizonzentrationen } \\
\hline & \multicolumn{2}{|c|}{$1 / 1$ n. $\left(C_{3}\right)_{2} S_{1} 1 / 4$ n. KCNS 1$)$} & \multicolumn{2}{|c|}{$1 / 2$ n. $\left(\mathrm{CH}_{3} \mathrm{l}_{\mathrm{S}} \mathrm{SO}_{4} 1 / 4\right.$ n. KCNS 1$)$} & \multicolumn{2}{|c|}{$1 / 6$ n. $\left(\mathrm{CH}_{3}\right)_{2} \mathrm{SO}_{4} 1 / 8$ n. $\left.K C N S 2\right)$} & \multicolumn{2}{|c|}{$1 / 1$, n. $\left(\mathrm{CH}_{g}\right)_{2} \mathrm{SO}_{4} 1_{2}$ n. NaCNS 1$)$} \\
\hline & $a$ & $\boldsymbol{K}$ & $a$ & $\boldsymbol{K}$ & $a$ & $K$ & $a$ & $K$ \\
\hline $\begin{array}{r}0 \\
5 \\
10 \\
15 \\
30 \\
60 \\
120 \\
180 \\
210 \\
\infty\end{array}$ & $\begin{array}{c}4,60 \mathrm{ccm} \\
3,72 ", \\
3,10 " \\
2,70 " \\
2,20 " \\
1,70 " \\
\text { - } \\
1,10 " \\
0,60 "\end{array}$ & $\begin{array}{l}- \\
0,095 \\
0,090 \\
0,082 \\
0,058 \\
0,040 \\
\overline{-} \\
0,017\end{array}$ & $\begin{array}{l}4,70 \mathrm{ccm} \\
4,10 " \\
3,40 " \\
3,07 " \\
2,20 " \\
1,50 " \\
1,10 " \\
0,95 " \\
0,40 "\end{array}$ & $\begin{array}{l}-\overline{120} \\
0,149 \\
0,134 \\
0,128 \\
0,103 \\
0,069 \\
0,05^{2} \\
-\end{array}$ & $\begin{array}{c}6,08 \mathrm{ccm} \\
6,00 " \\
5,88 " \\
5,80 " \\
5,52 " \\
5,00 " \\
= \\
= \\
2,90 "\end{array}$ & $\begin{array}{c}- \\
0,021 \\
0,028 \\
0,026 \\
0,027 \\
0,028 \\
- \\
- \\
-\end{array}$ & $\begin{array}{c}9,20 \mathrm{ccm} \\
7,80 \quad " \\
6,65 " \\
5,80 " \\
4,50 " \\
3,60 " \\
2,80 \text { " } \\
\text { - } \\
1,20 "\end{array}$ & $\begin{array}{c}-\overline{0} \\
0,064 \\
0,070 \\
0,068 \\
0,056 \\
0,038 \\
0,025 \\
= \\
-\end{array}$ \\
\hline
\end{tabular}

r) $n=1 / 20$ n, $v=2 \mathrm{ccm}$. a) $n=1 / 20 \mathrm{n}$., $v=5 \mathrm{ccm}$.

$$
K=\frac{I}{0,4343 a_{\infty} t} \log \frac{\left(a_{0}-a_{\infty}\right) a_{n}}{a_{0}\left(a_{n}-a_{\infty}\right)} \cdot \frac{v}{n}
$$

in welcher der Faktor $\frac{v}{n}$ zur Ueberfahrung der

Konstante auf dieselben Einheiten wie zuvor (Mole pro Liter) dient. Nach dieser Gleichung sind die folgenden Konstanten berechnet worden.

Die $K$. Werte halten sich innerhalb jeder Versuchsreibe sehr schön konstant; die in der Tabelle 6 angegebenen Mittelwerte stimmen gut mit den Konstanten äberein, welche fär àquivalente Losungen von gleicher Konzentration gefunden wurden (Tabelle 3,4 und 5). Letztere sind zum bequemeren Vergleich nochmals in der Tabelle 6 in Klammern beigefagt worden.

Ein ganz anderes Bild liefern diejenigen Versuche, in welchen ein UeberschuB des Dimethylsulfats zur Anwendung kam. Die "Geschwindigkeitskonstante" ist innerhalb jeder Versuchsreihe nicht mehr konstant, sondern nimmt stetig ab (besonders gegen Ende der Reaktion); ihr Wert ist uberhaupt bedeutend geringer, als in den entsprechenden Gemischen, in welchen beide Stoffe in a quivalentem Verhăltnis vorhanden waren, oder in welchen das Salz im Ueberschuß angewandt war. Das Ergebnis lautet also kurz dahin, daB ein UeberschuB des Dimethylsulfats die Reaktion verzőgert. Einige Beispiele mögen zur Veranschaulichung dieser Tatsache dienen.

Kommen auf I Mol des Salzes $\alpha$ Mole des Esters zur Einwirkung, dann ist:

$$
a=\alpha b \text {. }
$$

Ersetzt man weiter, wie fruher:

$$
b=a_{o} \cdot \frac{n}{v} \text { und } x=\left(a_{o}-a_{n}\right) \frac{n}{v} \text {, }
$$

so liefert die Forınel (2) für die Berechnung der Grobe $K$ den Ausdruck:

$K=\frac{\mathrm{I}}{0,4343(\alpha-\mathrm{I}) a_{0} t} \cdot \frac{v}{n} \log \frac{(\alpha-\mathrm{I}) a_{0}+a_{n}}{\alpha a_{n}}(4)$

Nach dieser Formel sind die Konstanten. der Tabelle 7 berechnet worden. Ein Blick auf diese Tabelle lehrt uns aber, daß die "Konstanten" nur am Anfang einer jeden Versuchsreihe wirklich konstant sind; später nehmen sie rasch ab. Aber auch die höchsten $K$-Werte sind immerhin noch bedeutend niedriger, als bei aquivalenten Lösungen. In der ersten Spalte sinkt die Konstante sogleich auf $1 / 3$, in der zweiten auf $1 / 2$ und in der dritten bis auf ungefähr $1 / 10$ ihres Normalwertes!

\section{Der Temperaturkoeffizient}

der Geschwindigkeitskonstante ist in einigen Fällen experimentell bestimmt worden. Für den EinfluB der Temperatur hat van't Hof ${ }^{1}$ ) eine empirische Beziehung gefunden, welche sich der Erfahrung ganz gut anschlieBt:

$$
\log K=a+b t
$$

Für ein Intervall von Io 0 erhält er dann den Quotienten der Konstanten:

$$
\frac{K_{t}+\text { 10 }}{K_{t}}={ }_{10}^{10 b}=k
$$

\begin{tabular}{|c|c|c|c|}
\hline $\begin{array}{c}\text { Tempe- } \\
\text { ratur } \\
\text { Grad }\end{array}$ & Anfangskonzentrationen & $K_{t}$ & $\begin{array}{c}k= \\
\frac{K_{t-10}}{K_{t}}\end{array}$ \\
\hline $\begin{array}{r}0 \\
25\end{array}$ & \}$_{1 / 1}$ n. $\left(\mathrm{CH}_{3}\right)_{2} S O_{4} 1 / 1$ n. $N a J_{1}^{f}$ & $\begin{array}{l}0,0298 \\
x, 04\end{array}$ & 4,14 \\
\hline$\stackrel{0}{12,6}$ & $\left\{1 / 8\right.$ n. $\left(C H_{3}\right)_{8} S O_{4} \quad 1 / 2$ n. $N a J$ & $\begin{array}{l}0,0295 \\
0,142\end{array}$ & $3,4^{8}$ \\
\hline $\begin{array}{c}0 \\
12,6\end{array}$ & $1 / 1 / 4$ n. $\left(\mathrm{CH}_{3}\right)_{8} \mathrm{SO}_{4} \quad 1 / 4$ n. $\mathrm{NaJ}$ & $\begin{array}{l}0,0,320 \\
0,140\end{array}$ & 3,23 \\
\hline $\begin{array}{l}25 \\
40\end{array}$ & $f_{1 / 4}$ n. $\left(C_{3}\right)_{2} S_{4} 1 / 4$ n. KCNS & $\begin{array}{l}0,236 \\
1,01\end{array}$ & 2,64 \\
\hline $\begin{array}{l}25 \\
40\end{array}$ & $\left.f_{1 / 8} \mathrm{n} \cdot\left(\mathrm{CH}_{3}\right)_{2} \mathrm{SO}_{4} 1 / 2 \mathrm{n} \cdot \mathrm{NaCNS}\right\}$ & $\begin{array}{l}0,196 \\
0,804\end{array}$ & 2,56 \\
\hline
\end{tabular}

In den meisten Fallen ist dieser Quotient, $k$, zwischen 2 und 3 eingeschlossen.

Tabelle 8.

Lösungsmittel: Methylalkohol.

I) van't $H$ of $f$, Vorlesungen über theoretische und physikalische Chemie 1, 224. Braunschweig 1898 . 
Wie aus dieser Tabelle zu ersehen ist, werden auch in unserem Falle ahnliche Zahlen erhalten.

\section{Versuthe in Aceton}

sind nur in sparlicher $\boldsymbol{Z}_{\text {ahl }}$ ausgefahrt worden. Ein Hindernis lag in dem geringeren Losungsvermögen des Acetons gegenuber den Salzen, und insbesondere den Rhodaniden. Aus dem Reaktionsgemisch kristallisiert das methylschwefelsaure Salz heraus, und dieser Umstand erschwert die Herausnahme der Proben.

Tabelle 9 .

Lösungsmittel: Aceton.

\begin{tabular}{|c|c|c|c|}
\hline $\begin{array}{c}\text { Tempe- } \\
\text { ratur } \\
\text { Grad }\end{array}$ & Anfangskonzentrationen & $\begin{array}{c}K^{\prime} \\
\text { (in } \\
\text { Aceton) }\end{array}$ & $\begin{array}{c}{ }_{(\text {in }}^{K} \\
\left.C H_{\mathrm{g}} O H\right)\end{array}$ \\
\hline $\begin{array}{c}0 \\
12,6 \\
0\end{array}$ & 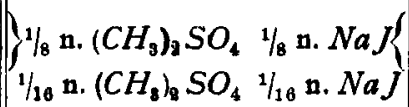 & $\begin{array}{c}3,36 \\
17,9 \\
4,75\end{array}$ & $\begin{array}{l}(0,0389) \\
(0,170) \\
(0,0377)\end{array}$ \\
\hline
\end{tabular}

Die Konstante in Aceton abertrifft die Konstante in Methylalkohol (welche in Klammern beigefagt ist) fast um das Hundertfache! Dieses ist um so mehr befremdend, als die Dielektrizitatskonstante, auf deren Zusammenhang mit der Reaktionsgeschwindigkeit von mehreren Forschern 1) hingewiesen wurde, beim Aceton viel kleiner als beim Methylalkohol ist:

$$
\begin{array}{ll}
\text { Aceton } & \varepsilon_{20}=20,7, \\
\text { Methylalkohol } & \varepsilon_{20}=32,5 .
\end{array}
$$

Dementsprechend ist auch die dissoziierende Kraft des Methylalkohols größer, als diejenige des Acetons, was aus Leitfahıgkeitsmessungen am $\left(C_{2} H_{5}\right)_{4} J$ hervorgeht $\left.{ }^{2}\right)$. Wir haben hier also mit einer bemerkenswerten Ausnahme von dem Parallelismus zwischen Dielektrizitätskonstante und Reaktionsgeschwindigkeit zu tun. Im Zusammenhang damit sei noch erwahnt, dab auch das Gleichgewicht in Aceton ein ganz anderes ist: die Reaktion geht in Aceton fast bis zu Ende, was allerdings auch durch die Schwerloslichkeit des Reaktionsproduktes begunstigt wird.

Der Temperaturquotient für 100 ist nicht wesentlich verschieden: er wird berecbnet $z u$ : 3,78 (im Methylalkohol: 3,35 im Durchschnitt).

\section{Andere Salze.}

Außer Natriumjodid und den Rhodaniden des Kaliums und des Natriums sind noch einige andere Salze in den Kreis der Untersuchung mit hereingezogen worden, teils um den Einflub des Anions, hauptsächlich aber um den

I) Vergl. die Zusammenstellung und Kritik von A. Michael, Berl. Ber. 41, 1080 (1908).

2) P. Walden, Zeitschr. f. physik. Chemie 54, 129 1905).
Einfluß des positiven Ions auf die Reaktionsgeschwindigkeit kennen zu lernen.

Von den Anionen kam nur das $B r$-Ion zur Anwendung, und zwar in Gestalt des Kalium. bromids. Dieses Salz lost sich jedoch in Methylalkohol ziemlich schwer auf, so dath nur ganz verdannte Lossungen untersucht werden konnten.

Tabelle 10.

$\left(\mathrm{CH}_{3}\right)_{2} \mathrm{SO}_{4}+\mathrm{KBr}=\mathrm{CH}_{3} \mathrm{Br}+\mathrm{K} \cdot \mathrm{CH}_{3} \mathrm{SO}_{4}$. Lösungsmittel: Methylalkohol.

Anfangskonzentrationen: $1 / 10$ n. $\mathrm{KBr}+1 / 10$ n. $\left(\mathrm{CH}_{3}\right)_{2} \mathrm{SO}_{4}$.

\begin{tabular}{|c|c|c|}
\hline$t$ & $a$ & $\begin{array}{c}K \\
\text { (nach Gleichung I) }\end{array}$ \\
\hline$o^{\prime}$ & $10,31 \mathrm{ccm}$ & - \\
\hline $3^{\prime}$ & 8,88 " & $(0,182)$ \\
\hline $45^{\prime}$ & $8,57 "$ & 0,157 \\
\hline $80^{\prime}$ & 7,85, & 0,147 \\
\hline $\mathbf{I} \mathbf{5}^{\prime}$ & 7,27, & $0, I_{4} 8$ \\
\hline $170^{\circ}$ & $6,60 "$ & 0,151 \\
\hline$\infty$ & 3,80, & - \\
\hline
\end{tabular}
Temperatur: $25^{\circ}, n-1 / \mathrm{s} 0^{\circ} \quad v=10 \mathrm{ccm}$.

Nimmt man an, daß die Geschwindigkeit der Einwirkung des Jodkaliums von derjenigen des Jodnatriums nicht wesentlich verschieden ist (was nach Analogie mit den Rhodaniden als durchaus wahrscheinlich erscheinen muß), und extrapoliert man letzteren Wert auf $25^{\circ}$ mit Hilfe des mittleren Temperaturquotienten: 3,35 , so erhalt man folgende Reibenfolge for die Reaktionsfahigkeit der drei untersuchten $A$ nionen bei $25^{\circ}$ und in etwa $1 / 16 \mathrm{n}$. Lơsung:

$$
J^{\prime}: 0,853 \rightarrow C N S^{\prime}: 0,299 \rightarrow B r^{\prime}: 0,157 \text {. }
$$

Von den untersuchten Anionen ist also das Jodion das reaktionsfăhigste. Parallel damit geht auch der Prozentsatz der Umsetzung: wahrend beim Natriumjodid die umgesetzte Menge mehr als $90 \%$ beträgt, geht die Reaktion bei den Rhodaniden nur bis 75 bis $80 \%$ und macht bej der Einwirkung des Kaliumbromids schon bei $6_{3} \%$ halt.

Noch deutlicher tritt der EinfluB des Kations auf die Reaktionsgeschwindigkeit zutage.

Tabelle II.

\begin{tabular}{|c|c|c|c|c|c|}
\hline \multicolumn{2}{|c|}{$1 / 4\left(\mathrm{CH}_{8}\right)_{8} \mathrm{SO}_{6}$} & \multirow{2}{*}{$\frac{1 / 6 \frac{S n J_{d}}{4}}{K}$} & \multicolumn{2}{|c|}{${ }_{1 / 8}\left(\mathrm{CH}_{\mathrm{g}}\right)_{2} \mathrm{SO}_{4}$} & \multirow{2}{*}{$\frac{1 / 8 \frac{S n J_{4}}{4}}{K}$} \\
\hline$t$ & $a$ & & $t$ & $a$ & \\
\hline $\begin{array}{l}0^{\prime} \\
60^{\prime} \\
120^{\prime} \\
180^{\prime} \\
240^{\prime} \\
300^{\prime} \\
\infty\end{array}$ & $\begin{array}{c}12,47 \mathrm{ccm} \\
9,95 " \\
8,50 " \\
8,13 " \\
7,60 " \\
7,25 " \\
5,15 "\end{array}$ & $\begin{array}{c}- \\
0,0702 \\
0,0792 \\
0,0649 \\
0,0664 \\
0,0665 \\
-\end{array}$ & $\begin{array}{c}0^{\prime} \\
30^{\prime} \\
60^{\prime} \\
90^{\prime} \\
120^{\prime} \\
33^{\prime} \\
\infty\end{array}$ & $\begin{array}{l}6,13 \\
5,50 \quad " \\
5,20 \quad " \\
4,87 \quad " \\
4,73 \quad " \\
3,63 \quad " \\
2,53 \quad "\end{array}$ & $\begin{array}{c}-\overline{-} \\
0,115 \\
0,0947 \\
0,0976 \\
0,0865 \\
0,112 \\
-\end{array}$ \\
\hline
\end{tabular}

$\underset{4}{4\left(\mathrm{CH}_{3}\right)_{8} \mathrm{SO}_{4}+\mathrm{SnJ}_{4}={ }_{4} \mathrm{CH}_{3} J+\underset{\text { Temperatur: }}{\mathrm{Sn}\left(\mathrm{CH}_{3} \mathrm{SO}_{4}\right)_{4}} .}$ $v=5 \mathrm{ccm} . \quad n=1 / 20^{\circ}$ 
Zinnjodid reagiert mit Methylsulfat langsamer als Natriumjodid. Die Umsetzung geht etwas mehr als zur Halfte. Die Konstanten sind nach der Gleichung ( $\mathrm{I}$ ) berechnet worden. Ihre Uebereinstimmung in jeder Versuchsreihe deutet darauf hin, daf auch hier die Reaktion sich bimolekular abspielt, während die Reaktionsformel eine quinquimolekulare Reaktion erwarten labt. Es ist jedoch bekannt, daß derartige "Vereinfachungen" des Reaktionsicrlaufes keineswegs zu den Seltenheiten gehören ${ }^{1}$ ). Im übrigen scheint jedoch die Konstante mit der Konzentrationszunahme der Lösung noch stärker abzunehmen als beim Natriumjodid (Tabelle 3).

- Noch langsamer reagiert Kadmiumjodid:

Tabelle 12.

$2\left(\mathrm{CH}_{8}\right)_{9} \mathrm{SO}_{4}+\mathrm{CdJ}_{\mathrm{a}}-2 \mathrm{CH}_{3} \mathrm{~J}+\mathrm{Cd}\left(\mathrm{CH}_{3} \cdot \mathrm{SO}_{4}\right)_{2}$. Lösungsmittel: Methylalkohol. $v=5 \mathrm{ccm} . \quad n=1 / 80^{\circ}$

\begin{tabular}{|c|c|c|c|c|c|}
\hline \multirow{2}{*}{$\begin{array}{c}\text { Tompe- } \\
\text { ratur } \\
25^{\circ} \\
1\end{array}$} & \multicolumn{2}{|c|}{$\begin{array}{l}\text { Anfangskonzentration } \\
1 / 8\left(C \dot{H}_{3}\right)_{2} S O_{6}+1 / 8 \frac{C d J_{2}}{2}\end{array}$} & \multirow{2}{*}{$\begin{array}{c}\text { Tempe- } \\
\text { ratur } \\
40^{\circ} \\
t\end{array}$} & \multicolumn{2}{|c|}{$\begin{array}{l}\text { Anfangskonzentration } \\
1_{4}\left(\mathrm{CH}_{3}\right)_{2} \mathrm{SO}_{4}+1 / 4 \frac{\mathrm{Cd} J_{2}}{2}\end{array}$} \\
\hline & $a$ & $\boldsymbol{K}$ & & $a$ & $\boldsymbol{K}$ \\
\hline $\begin{array}{c}0^{\prime} \\
30^{\prime} \\
90^{\prime} \\
150^{\prime} \\
1260^{\prime} \\
\infty\end{array}$ & $\begin{array}{l}6,20 \mathrm{ccm} \\
6,15 " \\
6,05 " \\
5,98 " \\
5,60 " \\
5,50 "\end{array}$ & $\begin{array}{c}-\overline{0} \\
0,041 \\
0,049 \\
0,049 \\
0,076 \\
-\end{array}$ & $\begin{array}{r}0^{\prime} \\
30^{\prime} \\
150^{\prime} \\
210^{\prime} \\
300^{\prime} \\
\infty\end{array}$ & $\begin{array}{l}\text { I } 2,38 \mathrm{ccm} \\
\text { II,90 " } \\
\text { II,35 " } \\
\text { II,23 " } \\
\text { I I,05 " } \\
\text { II,05 " }\end{array}$ & $\begin{array}{c}- \\
0,152 \\
0,185 \\
0,245 \\
- \\
-\end{array}$ \\
\hline & \multicolumn{2}{|c|}{$K_{\text {mittel }}=0,054$} & \multicolumn{3}{|c|}{$K_{\text {mittel }}=0,194$} \\
\hline
\end{tabular}

Beim Kadmiumjodid ist die Reaktion sehr unvollstăndig, in Anbetracht dessen haben die Geschwindigkeitskonstanten, welche nach der Gleichung (I) berechnet wurden, nur einen annăhernden Wert. Sie sollen lediglich zum Vergleich mit anderen Salzen dienen. Der Vergleich ergibt far die Reihenfolge der Geschwindigkeit der Umsetzung der Jodide in $1 / 8 n$. Losung bei $25^{\circ}$ folgende Reihe:

$$
\begin{aligned}
\mathrm{NaJ}: 0,853 \rightarrow & \frac{\mathrm{SnJ}_{4}}{4}: 0, \text { roI } \rightarrow \frac{C d J_{2}}{2}: 0,054 . \\
& \text { Tabelle I3. }
\end{aligned}
$$

$\left(\mathrm{CH}_{8}\right)_{2} \mathrm{SO}_{4}+\mathrm{NH}_{4} \mathrm{CNS}=\mathrm{CH}_{3} \mathrm{CNS}+\mathrm{CH}_{3} \cdot \mathrm{NH}_{4} \mathrm{SO}_{4}$. Lösungsmittel: Methylalkohol. Temperatur: $25^{\circ}$.

\begin{tabular}{|c|c|c|c|c|c|}
\hline \multicolumn{3}{|c|}{$1 / 2 \mathrm{n} \cdot\left(\mathrm{CH}_{3}\right)_{2} \mathrm{SO}_{4}+1 / 2 \mathrm{n} \cdot \mathrm{NH}_{4} \mathrm{CNS}$} & \multicolumn{3}{|c|}{$1 / 4$ n. $\left(\mathrm{CH}_{3}\right)_{2} \mathrm{SO}_{4}+1 / 4$ n. $N H_{1} \mathrm{CNS}$} \\
\hline$t$ & $a$ & $K$ & $t$ & $a$ & $K$ \\
\hline \multirow{6}{*}{$\begin{array}{r}0^{\prime} \\
5^{\prime} \\
10^{\prime} \\
15^{\prime} \\
30^{\prime} \\
60^{\prime}\end{array}$} & $9,45 \mathrm{ccm}$ & - & \multirow{7}{*}{$\begin{array}{c}0^{\prime} \\
5^{\prime} \\
10^{\prime} \\
15 \\
30^{\prime} \\
60^{\prime} \\
\infty\end{array}$} & $4,80 \mathrm{ccm}$ & - \\
\hline & 8,45, & 0,156 & & \multirow{2}{*}{$\begin{array}{l}4,45 " \\
4,15\end{array}$} & 0,177 \\
\hline & 7,80 & 0,142 & & & 0,180 \\
\hline & 7,15, & 0,153 & & $\begin{array}{l}4, I 5 \quad " \\
3,90 \quad "\end{array}$ & 0,182 \\
\hline & $6, \infty$ & 0,157 & & $\begin{array}{ll}3,90 & " \\
3,35 & \end{array}$ & 0,183 \\
\hline & 4,90 & 0,160 & & $2,60, "$ & 0,211 \\
\hline \multirow{2}{*}{$\begin{array}{c}120^{\prime} \\
\infty\end{array}$} & 4,00 & \multirow[t]{2}{*}{0,175} & & I, I5 " & - \\
\hline & 2,90 & & & \multicolumn{2}{|c|}{$K_{\text {mittel }}=0,186$} \\
\hline & \multicolumn{2}{|c|}{$K_{\text {mittel }}=0,157$} & & & \\
\hline
\end{tabular}

$$
n=1 / 20^{\circ} \quad v=2 \mathrm{ccm} \text {. }
$$

I) van't Hoff-Cohen, Studien zur chemischeu Dynamik, S. 104 ( 1896$)$.
Hingegen ist der Temperaturquotient (für 10 ${ }^{0}$ ) der Reaktionskonstante des Kadmiumjodids von derjenigen des Natriumjodids nur wenig verschieden: er berechnet sich zu 2,35 (fär $\mathrm{NaJ}: 3,66$ ).

Von den Rhodaniden sind außer dem Kalium- und Natriumsalz noch Ammoniumrhodanid und Kobaltrhodanid untersucht worden.

Tabelle I4.

$$
\begin{gathered}
2\left(\mathrm{CH}_{3}\right)_{2} \mathrm{SO}_{4}+\mathrm{Co}\left(\mathrm{CNS}_{2}\right. \\
=2 \mathrm{CH}_{3} \cdot \mathrm{CNS}+\mathrm{Co}\left(\mathrm{CH}_{3} \cdot \mathrm{SO}_{4}\right)_{2} .
\end{gathered}
$$

Lösungsmittel: Methylalkohol. Temperatur: $25^{\circ}$. $v=2 \mathrm{ccm} . \quad n=1 / 20$.

Anfangskonzentration: ${ }_{1 / 2}$ n. $\left(\mathrm{CH}_{3}\right)_{4} \mathrm{SO}_{4}+1 / 4$ n. $\mathrm{Co}(\mathrm{CNS})_{8}$.

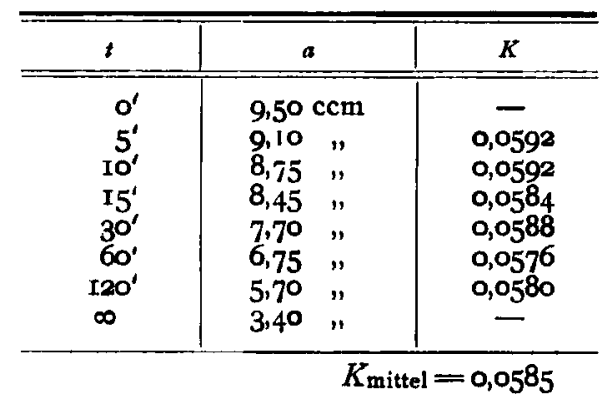

Vergleichen wir alle Rhodanide untereinander, so erhalten wir folgende Reihenfolge far $1 / 2 \mathrm{n}$. Losungen und $25^{\circ}$ :

$$
K C N S: 0,224 \rightarrow N a C N S: 0,196
$$

$$
\rightarrow \mathrm{NH}_{4} \mathrm{CNS}: 0, \mathrm{I} 57 \rightarrow \mathrm{Co}(\mathrm{CNS})_{2}: 0,05^{8} \text {. }
$$

Auch hier tritt ein deutlicher Parallelismus der Reaktionskonstanten mit der Verschiebung des Gleichgewichtes zutage: je vollstandiger die Reaktion verläuft, um so grobler ist ihre Geschwindigkeit; das zeigt sich deutlich beim Vergleich der Endtiter $1 / 2$ n. Losungen, und zwar:

$K C N S:$ 1,70 $\mathrm{ccm} \rightarrow N a C N S: 1,78 \mathrm{ccm}$ $\rightarrow N H_{4} C N S: 2,90 \mathrm{ccm} \rightarrow \operatorname{Co}(C N S)_{2}: 3,40 \mathrm{ccm}$.

Ueber den Zusammenhang des Gleichgewichtes und der Reaktionskonstanten mit dem Dissoziationsgrade der reagierenden Stoffe sollen weitere Untersuchungen Aufschluß bringen.

\section{Zusammenfassung.}

I. Die Reaktion zwischen Alkylsulfaten und Salzen in methylalkoholischer (bezw. Aceton-) Lősung führt zu einem Gleichgewichtszustand.

2. Der Verlauf der untersuchten Reaktionen ist bimolekular.

3. Ein UeberschuB des Dimethylsulfats verlangsamt die Reaktion.

4. Der Temperaturquotient der Reaktionskonstanten fär Io ${ }^{0}$ liegt zwischen 2,6 und 3,7 .

5. In Aceton geht die Reaktion hundertmal schneller als in Methylalkohol. 
6. Die Reaktionsgeschwindigkeit hångt von der Natur beider Ionen des angewandten Salzes ab.

7. Je vollstăndiger die Reaktion verläuft, um so gröber ist ihre Geschwindigkeit.
8. Nur eine Methylgruppe ist im Dimethylsulfat gegen Metalle ersetzbar.

Riga, Physikalisch-chemisches Laboratorium des Polytechnikums, I8./3 I. Mărz r9o9.

(Eingegangen: 6. April.)

\title{
ÜBER DIE KATHODISCHE ZERSTÄUBUNG VON METALLEN IN VERDUNNTEN GASEN. V. MITTEILUNG.
}

\author{
Die Zerstäubungsreihe der Metalle und der EinfluB des Gases \\ auf die Zerstaubbarkeit. \\ Von $V$. Kohlschitter.
}

\section{Einleitung.}

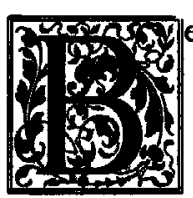
ei den letzten Erorrterungen aber die kathodische Zerstăubung, die in der Z. f. Elektroch. stattgefunden haben und sich an frahere Mitteilungen von mir und meinen Mitarbeitern anschlossen ${ }^{1}$ ), handelte es sich einesteils um eine Deutung des ganzen Vorganges, anderenteils um den Einfluß verschiedener Gase auf die Zerstäubbarkeit des gleichen Metalles.

Ueber den ersteren, rein theoretischen Punkt haben F. Fischer und J. Stark Ansichten geăußert, mit denen beide Forscher zwar tatsachlich weit auseinandergehen, sich aber unter dem Stichwort "physikalische Theorie der Zerstäubungserscheinungen" darin begegnen, daß sie die von mir fruher entwickelten chemischen Vorstellungen ablehnen. Dieser Punkt soll far die folgende Mitteilung außer Betracht bleiben, da ich ihn demnåchst an anderer Stelle zu besprechen gedenke.

Die $z$ weite Frage ist eine experimentelle und als solche von mir und meinen Mitarbeitern auf der einen, von Fischer und Hähnel auf der anderen Seite in entgegengesetztem Sinne beantwortet worden. Wahrend wir eine starke Zunahme der Zerstäubung mit dem Atomgewicht des Gases beobachteten, erklarten Fischer und $\mathrm{Hahhnel}$ auf Grund ihrer Versuche diesen Befund zunächst als durch mangelhafte Versuchsanordnung veranlaBit, und sahen auch spăter, nachdem ich die fraheren Beobachtungen durch neue, auch in ihrem Sinne einwandfreie, Versuche beståtigt hatte, die gleiche Zerstaubbarkeit in den verschiedenen Gasen als das Primare, die Verschiedenheit als durch besondere Versuchsbedingungen, insbesondere hohe Belastung der Rohren, herausgearbeitet, an.

I) V. Koblschutter und R. Muller, Z. f. Elektroch. 12, 365 (1906); V. K o h l s c h t t t e r, ebenda 12, 869 (I906); V. Kohlschutter und Th. Goldschmidt, ebenda 14, 221 (1908); F. Fischer und O. Hähnel, ebenda 14, 366, 433,677 (I908); V. Kohlschâtter, ebenda 14, 417 [4. Mitteilung], 437, 68r (1908); B. Walter, ebenda 14, 695 (1908); J. Stark, ebenda 14, $75^{2}$ (1908).
Die Diskussion konnte damals zu keinem sachlichen AbschluB gebracht werden, da beide Teile auf die Verwendung von Induktorien als Stromquelle angewiesen waren und die elektrischen Bedingungen daher nicht hinreichend vergleichbar definiert werden konnten. Eine erneute Prufung der Frage unter Anwendung konstanter Strome war daher erforderlich; sie bildet den einen Gegenstand der vorliegenden Mitteilung.

Neben der Abhăngigkeit von der Natur der Gase ist far die Beurteilung der ganzen Erscheinung die Reihenfolge der Metalle nach ihrer Zerstäubbarkeit von entscheidender Bedeutung. Die wichtigste Untersuchung aber diese Frage, mit der sich schon (rookes ${ }^{1}$ ) und Granquist ${ }^{2}$ ) beschäftigt haben, rährt von Holborn und Austin ${ }^{3}$ ) her. Diese fanden, dab die Mengen verschiedener Metalle, die unter gleichen Bedingungen zerstäuben, in einfachen Beziehungen $z \mathrm{u}$ ihren Atomgewichten stehen, und zeigten, daB auch das Material von Granquist sich dieser GesetzmåBigkeit unterordnen läft.

Wegen der fundamentalen Wichtigkeit des Resultates schien es mir geboten, diese Versuche wenigstens so weit $\mathrm{zu}$ wiederholen, daB von mir aus ein sicherer Anschlub an sie hergestellt wärde, nachdem ich schon mit Goldschmidt einen solchen vorlaufig $z u$ erreichen versucht hatte. In gewissem Sinne war auch eine Nachprufung wünschenswert. Die Versuche von Granquist und Holborn und Austin sind in Luft ausgefuhrt; es kam also nicht nur kein einheitliches Gas zur Verwendung, sondern das Gemenge anderte auch noch wahrend des Versuches seine Zusammensetzung, da Sauerstoff durch das zerstäubte Metall absorbiert und der Druck durch Nachfüllen von Luft konstant gehalten wurde. Da zudem die in Luft beob.

I) Crookes, Proc. Roy. Soc. 50, 88 (189r).

2) Granquist, Oefvers. af Svensk. Vetensk. Akad. Förhand1. 1898, S. 709 .

3) Holborn und A stin, Wissenschaftl. Abhandl. der Physik.-Techn. Reichsanstalt 4, IOI. 